\title{
Correction to: The Perfect Moral Storm: Diverse Ethical Considerations in the COVID-19 Pandemic
}

\author{
Vicki Xafis ${ }^{1} \cdot$ G. Owen Schaefer ${ }^{1}$ - Markus K. Labude ${ }^{1} \cdot$ Yujia Zhu $^{1}$ • \\ Li Yang Hsu ${ }^{2,3}$
}

Published online: 6 June 2020

(C) National University of Singapore and Springer Nature Singapore Pte Ltd. 2020

\section{Correction to: Asian Bioethics Review https://doi.org/10.1007/s41649-020-00125-3}

Regrettably, in the original version of this article the name of one of the authors was spelt incorrectly. "Li Yan Hsu" should be "Li Yang Hsu". Li Yang Hsu is affiliated with the Saw Swee Hock School of Public Health, National University of Singapore, Singapore AND the Yong Loo Lin School of Medicine, National University of Singapore, Singapore.

Vicki Xafis

vicki.xafis@nus.edu.sg

1 SHAPES Initiative, Centre for Biomedical Ethics, Yong Loo Lin School of Medicine, National University of Singapore, Singapore

2 Saw Swee Hock School of Public Health, National University of Singapore, Singapore

3 Yong Loo Lin School of Medicine, National University of Singapore, Singapore 